\title{
Systematic Literature Review and Meta-analysis: Real-World Mucosal Healing in Vedolizumab-Treated Patients with Crohn's Disease
}

\author{
Silvio Danese $\mathbb{D}^{1,},{ }^{1,2}$ Pravin Kamble $\mathbb{D}^{\circ},{ }^{3}$ Jin Yang, ${ }^{4}$ Jean-Gabriel Le Moine, ${ }^{5}$ Shahnaz Khan, ${ }^{4}$ \\ Emma Hawe, ${ }^{5}$ Christian Agboton $\mathbb{D}^{6},{ }^{6}$ Song Wang, ${ }^{3}$ and Peter M. Irving $\mathbb{\circledR}^{7,8}$ \\ ${ }^{1}$ Gastroenterology and Endoscopy, IRCCS Ospedale San Raffaele, Milan, Italy \\ ${ }^{2}$ University Vita-Salute San Raffaele, Milan, Italy \\ ${ }^{3}$ Takeda, Cambridge, Massachusetts, USA \\ ${ }^{4}$ RTI Health Solutions, Research Triangle Park, North Carolina, USA \\ ${ }^{5}$ RTI Health Solutions, Manchester, UK \\ ${ }^{6}$ Takeda, Zurich, Switzerland \\ ${ }^{7}$ Guy's and St Thomas' NHS Foundation Trust, London, UK \\ ${ }^{8}$ School of Immunology and Microbial Sciences, King's College London, London, UK \\ Correspondence should be addressed to Pravin Kamble; pravin.kamble@takeda.com
}

Received 27 October 2021; Accepted 22 January 2022; Published 16 February 2022

Academic Editor: Than Than Aye

Copyright (C) 2022 Silvio Danese et al. This is an open access article distributed under the Creative Commons Attribution License, which permits unrestricted use, distribution, and reproduction in any medium, provided the original work is properly cited.

\begin{abstract}
Background. Vedolizumab is a gut-selective monoclonal anti- $\alpha_{4} \beta_{7}$-integrin antibody approved for the treatment of adults with moderately to severely active Crohn's disease (CD). Aim. To conduct a systematic literature review and meta-analysis of published real-world studies examining mucosal healing $(\mathrm{MH})$ rates in patients with $\mathrm{CD}$ treated with vedolizumab in routine clinical practice. Methods. MEDLINE-, Cochrane-, and EMBASE-indexed publications from January 2014 to January 2020 and 2018-2019 conference abstracts were searched for real-world studies reporting MH-related outcomes in vedolizumab-treated adults with CD. A meta-analysis was conducted in $\mathrm{R}$ to generate pooled estimates of $\mathrm{MH}$. The primary analysis included studies reporting point estimates of $\mathrm{MH} /$ endoscopic remission as absence of ulcers/erosions and/or Simple Endoscopic Score for CD (SESCD) cut - points $<4$, at 6 and 12 months. Results. The systematic literature review included 36 studies, predominantly of antitumour necrosis factor-experienced patients. $\mathrm{MH}$ and endoscopic remission were the most frequently reported endpoints. $\mathrm{MH}$ rates were $10.1 \%-46.0 \%$ at 6 months (ten studies) and 21.2\%-62.5\% at 12 months (eight studies). Fifteen studies defining MH as absence of ulcers/erosions and/or SES-CD cut-points $<4$ were included for meta-analysis. Pooled MH rates for the primary analysis were $31.8 \%$ at 6 months (95\% confidence interval (CI): 25.6-38.3; five studies, $N=223$ ) and $33.4 \%$ at 12 months (95\% CI: 25.9-41.4; three studies, $N=151$ ). Conclusion. Approximately one-third of vedolizumab-treated patients with $\mathrm{CD}$ achieved $\mathrm{MH}$ at both 6 and 12 months in real-world clinical settings, despite utilisation in largely biologic-refractory patients. These findings confirm the effectiveness of vedolizumab for achieving $\mathrm{MH}$ in patients with $\mathrm{CD}$.
\end{abstract}

\section{Introduction}

Crohn's disease $(\mathrm{CD})$ is a chronic and progressive inflammatory bowel disease in which uncontrolled inflammation can lead to structural bowel damage, causing long-term debilitating complications that often require surgery [1-3]. Treat- ment goals have recently evolved from symptom-based management toward control of inflammation to prevent bowel damage and promote mucosal healing ( $\mathrm{MH}$; as assessed by objective measures such as endoscopy) [4-6]. $\mathrm{MH}$ is generally referred to as endoscopic remission, describing the absence of ulceration; however, instruments 
used to assess $\mathrm{MH}$ and measurable parameters used in definitions of $\mathrm{MH}$-related endpoints are reported using variable nomenclature and are incompletely validated $[7,8]$. Endoscopic evaluations of $\mathrm{MH}$ commonly utilise the Simple Endoscopic Score for CD (SES-CD), CD Endoscopic Index of Severity score, or absence of ulceration/deep ulceration with/without aphthae $[5,9]$. Achievement of $\mathrm{MH}$ is associated with improved long-term outcomes, including reduced risk of relapse, decreased hospitalisations, increased rates of steroid-free remission, and longer resection-free intervals [5, 10-15]. Thus, consensus guidelines for CD management, including STRIDE II, consider $\mathrm{MH}$ to be an important therapeutic goal that may help patients achieve meaningful and sustained improvements in quality of life $[8,16,17]$.

Although $\mathrm{MH}$ is associated with better long-term outcomes for patients, it is hard to achieve compared with other clinical treatment targets in CD [18]. For example, in the SONIC trial (biologic- and immunomodulator-naïve patients treated with either infliximab or azathioprine or both), only $53 \%$ of patients in clinical remission (CD Activity Index (CDAI) score < 150) achieved MH (absence of ulceration) [19]. In addition, clinical indices like CDAI, long used to evaluate CD treatment efficacy, have been criticised for being less reliable as indicators of mucosal inflammation $[8,20,21]$. CDAI scores analysed from three placebocontrolled trials of adalimumab, upadacitinib, and risankizumab were only moderately correlated with mucosal inflammation of the bowel assessed endoscopically using SES-CD scoring [22].

Vedolizumab is a gut-selective monoclonal anti- $\mathrm{a}_{4} \beta_{7^{-}}$ integrin antibody approved for the treatment of adults with moderate to severe CD [23-25]. The GEMINI 2 and GEMINI 3 phase 3 clinical trials established the efficacy of vedolizumab for achieving clinical remission in patients with moderately to severely active CD [26, 27]. Several studies have established the effectiveness of vedolizumab as an induction and maintenance treatment for CD in real-world settings [28-32].

$\mathrm{MH}$ was not assessed as a main endpoint in $\mathrm{CD}$ in the GEMINI clinical trial programme; clinical studies of $\mathrm{MH}$ have been conducted in smaller, selected patient cohorts (e.g., the prospective, phase $3 \mathrm{~b}$, open-label VERSIFY trial of patients with active CD [18] and a retrospective study of a subset of patients enrolled in the open-label extension phase of GEMINI studies (GEMINI LTS) [33]). Real-world studies assessing $\mathrm{MH}$ endpoints in vedolizumab-treated patients may achieve a larger sample size by including a broader range of patients and thus may provide a better understanding of the clinical effectiveness of vedolizumab among diverse patient populations treated in routine clinical practice.

This systematic literature review (SLR) and metaanalysis of real-world studies published in the last 7 years was conducted to examine and provide pooled estimates of the rates of $\mathrm{MH}$ in vedolizumab-treated patients with $\mathrm{CD}$.

\section{Methods}

2.1. Study Selection. This SLR was conducted according to the general recommendations of the Cochrane handbook for Systematic Reviews of Interventions and PRISMA guidelines [34, 35]. MEDLINE-, Cochrane-, and EMBASEindexed publications in English from January 2014 to January 2020 (Supplementary Tables 1-3) and conference abstracts from 2018 to 2019 (Supplementary Table 4) were searched for real-world studies reporting $\mathrm{MH}$ and related outcomes in vedolizumab-treated patients with $\mathrm{CD}$. Bibliographies of identified relevant SLRs and network meta-analyses were reviewed for additional studies. No contact with authors was made to identify additional studies.

Search terms included combinations of free text and medical subject headings used to denote CD, inflammatory bowel disease, vedolizumab, and real-world studies (e.g., observational, real-world, case-control, cohort, and registry studies) (Supplementary Tables 1-4). The identified records were reviewed according to prespecified inclusion/ exclusion criteria (Table 1) by one researcher, and $50 \%$ of the search results were reviewed by a second independent researcher. Studies with $<10$ patients and patients aged $<$ 18 years were excluded.

2.2. Data Extraction and Quality Assessment. One researcher used predefined parameters to extract all data, which were quality checked by a second researcher for accuracy. Key information obtained for each eligible study included author, year of publication, country/region, study design, sample size, study duration, vedolizumab dosing, and patient inclusion/ exclusion criteria. Patient characteristics included age, sex, smoking status, disease duration, disease behaviour and location, and prior treatment history. Detailed study data on $\mathrm{MH}$-related outcomes were also collected. Quality assessment of studies was undertaken using the National Institutes of Health Quality Assurance tools for cohort and case-control studies (Supplementary Table 5) [36].

2.3. Statistical Analyses. Studies identified during the SLR were assessed for inclusion in a meta-analysis. Studies that reported results on overlapping patient populations or used median time for the endpoint data, as well as studies that did not report on timepoints or an endpoint of interest, were excluded. A random effects meta-analysis was conducted using the meta package in $\mathrm{R}$, using the method of restricted maximum likelihood and double arcsine transformation to generate pooled estimates of the proportion of patients achieving $\mathrm{MH}$ out of the total number treated with vedolizumab, with 95\% confidence intervals (CIs). A sensitivity analysis was performed using the logit transformation. Heterogeneity was considered using Higgins's $I^{2}$, providing an estimate of the percentage of variation across studies that was due to heterogeneity. All analyses used the endpoint of $\mathrm{MH}$ or endoscopic remission, defined as absence of ulcers and/or erosions or SES-CD cut - points $<4$. The primary analysis included studies reporting point estimates at approximately 6 months and separately at approximately 12 months; the secondary analysis also included cumulative rates at 6 months and separately at approximately 12 months, and the tertiary analysis expanded the evidence base further to include point estimates, cumulative rates, and studies reporting data up to 12 months. 
TABLE 1: SLR study inclusion and exclusion criteria.

\begin{tabular}{|c|c|c|c|}
\hline Criteria & Included & \multicolumn{2}{|l|}{ Excluded } \\
\hline Population $^{\dagger}$ & $\begin{array}{l}\text { (i) Adult patients (aged } \geq 18 \text { years; no upper age restriction) } \\
\text { (ii) Diagnosed with CD or IBD, with CD reported separately }\end{array}$ & \multicolumn{2}{|c|}{$\begin{array}{l}\text { (i) Nonhuman } \\
\text { (ii) Paediatric patients }(<18 \text { years of age) } \\
\text { (iii) Diagnosed with IBDu or other } \\
\text { non-IBD conditions }\end{array}$} \\
\hline Interventions & (i) ENTYVIO, vedolizumab, or MLN0002 & \multicolumn{2}{|c|}{$\begin{array}{l}\text { (i) Interventions other than vedolizumab } \\
\text { (ENTYVIO) }\end{array}$} \\
\hline Comparators & (i) No restrictions ${ }^{\dagger}$ & \multicolumn{2}{|l|}{ (i) No restrictions } \\
\hline Outcomes $^{\ddagger}$ & $\begin{array}{l}\text { (i) Mucosal healing or endoscopic remission, including, but not } \\
\text { limited to, the following measures } \\
\text { (a) Resolution/disappearance of ulcers/absence of mucosal } \\
\text { ulceration (complete healing) assessed by ileocolonoscopy } \\
\text { or MRI/MaRIA score/MRE-CGHAS and IGHAS scores } \\
\text { (b) CDEIS score (varying thresholds) } \\
\text { (c) Lewis score }<135 \text { (normal mucosa) } \\
\text { (d) Saverymuttu score } \\
\text { (e) Dieleman score } \\
\text { (f) Monitr }{ }^{\mathrm{TM}} \text { test (biomarkers) } \\
\text { (ii) Endoscopic improvement or endoscopic response } \\
\text { (a) Reduction from baseline in SES-CD score of }>50 \% \\
\text { (b) SES-CD score (varying thresholds) } \\
\text { (c) Rutgeert's scale } \leq 1\end{array}$ & \multicolumn{2}{|c|}{$\begin{array}{l}\text { (i) Any studies not providing detail on } \\
\text { any of the specific outcomes of interest } \\
\text { (ii) Safety-only studies }\end{array}$} \\
\hline Study design & $\begin{array}{l}\text { (i) Observational/real-world studies (prospective or retrospective, } \\
\text { case-control, cohort, or registries) } \\
\text { (ii) SLRs and NMAs were included at level } 1 \text { review to be } \\
\text { used for identification of primary studies (they were excluded } \\
\text { during level } 2 \text { review) }\end{array}$ & $\begin{array}{l}\text { (i) Clinical trials } \\
\text { (ii) Cross-sectional studies } \\
\text { (iii) Case reports } \\
\text { (iv) Editorials }\end{array}$ & $\begin{array}{l}\text { (i) Reviews } \\
\text { (ii) Notes } \\
\text { (iii) Comments } \\
\text { (iv) Letters }\end{array}$ \\
\hline Restrictions & $\begin{array}{l}\text { (i) English language } \\
\text { (ii) Date limit: January 1, 2014, to January 29, } 2020\end{array}$ & \multicolumn{2}{|l|}{ (i) Non-English language } \\
\hline
\end{tabular}

\section{Results}

3.1. Qualitative Summary: MH-Related Outcomes. Screening of 1751 potentially relevant records identified 36 vedolizumab real-world studies for inclusion in the SLR for qualitative evaluation (PRISMA flowchart in Figure 1 details publication screening and the reasons for exclusion). Most studies in the SLR were single-arm, retrospective cohort studies conducted in a single country (10/36 from the United States). Population size ranged from 13 to 650 patients, median patient age from 29.0 to 49.5 years, and median disease duration from 2.4 to 19.5 years (Supplementary Table 6). Most studies included a mix of both antitumour necrosis factor (TNF-) naïve and -experienced patients $(23 / 36)$.

$\mathrm{MH}$ was assessed by various instruments across studies, including endoscopy, magnetic resonance imaging, and computed tomography. For MH-related endpoints, 22 studies reported $\mathrm{MH}$, nine endoscopic remission, six endoscopic response, four endoscopic improvement, four deep remission, and two endoscopic healing, and a single study each reported endoscopic remission/response, radiologic improvement, radiologic remission, objective response, and objective remission based on endoscopic/radiographic assessment. Although definitions applied to these endpoints varied across studies, the majority defined $\mathrm{MH} /$ endoscopic remission as the absence of ulcers or erosions (17 studies) and/or a SES-CD score of $\leq 4$ (six studies).

Real-world $\mathrm{MH}$ rate ranges were $20.0 \%-22.2 \%$ at 3 months (mean $\pm \mathrm{SD}, 21.3 \% \pm 1.2 \%$; three studies [37-39]), $10.1 \%-46.0 \%$ at 6 months (mean $\pm \mathrm{SD}, 30.4 \% \pm 11.8 \%$; ten studies [29, 30, 39-46]), and $21.2 \%-62.5 \%$ at 12 months (mean \pm SD, 37.8\% $\pm 14.6 \%$; eight studies [28, 37, 39, 43, 47-51]). Cumulative 12-month $\mathrm{MH}$ rates were $54 \%-62.5 \%$ in anti-TNF-naïve patients (two studies $[28,49]$ ) and 47\%$59.7 \%$ in anti-TNF-experienced patients [28, 49].

Rates of endoscopic remission (absence of ulcers/erosions and SES-CD scores of $<4$ ) were reported in seven studies [52-58]. Rates of endoscopic remission in vedolizumabtreated patients were $15 \%-33 \%$ at/within 6 months (two studies $[54,57,58]$ ) and $27 \%-38 \%$ at or within 12 months (four studies $[53,56-58])$, with two studies contributing cumulative data over 6 months [54] and 6-12 months [58], respectively. The first of these [54] reported a significantly higher 


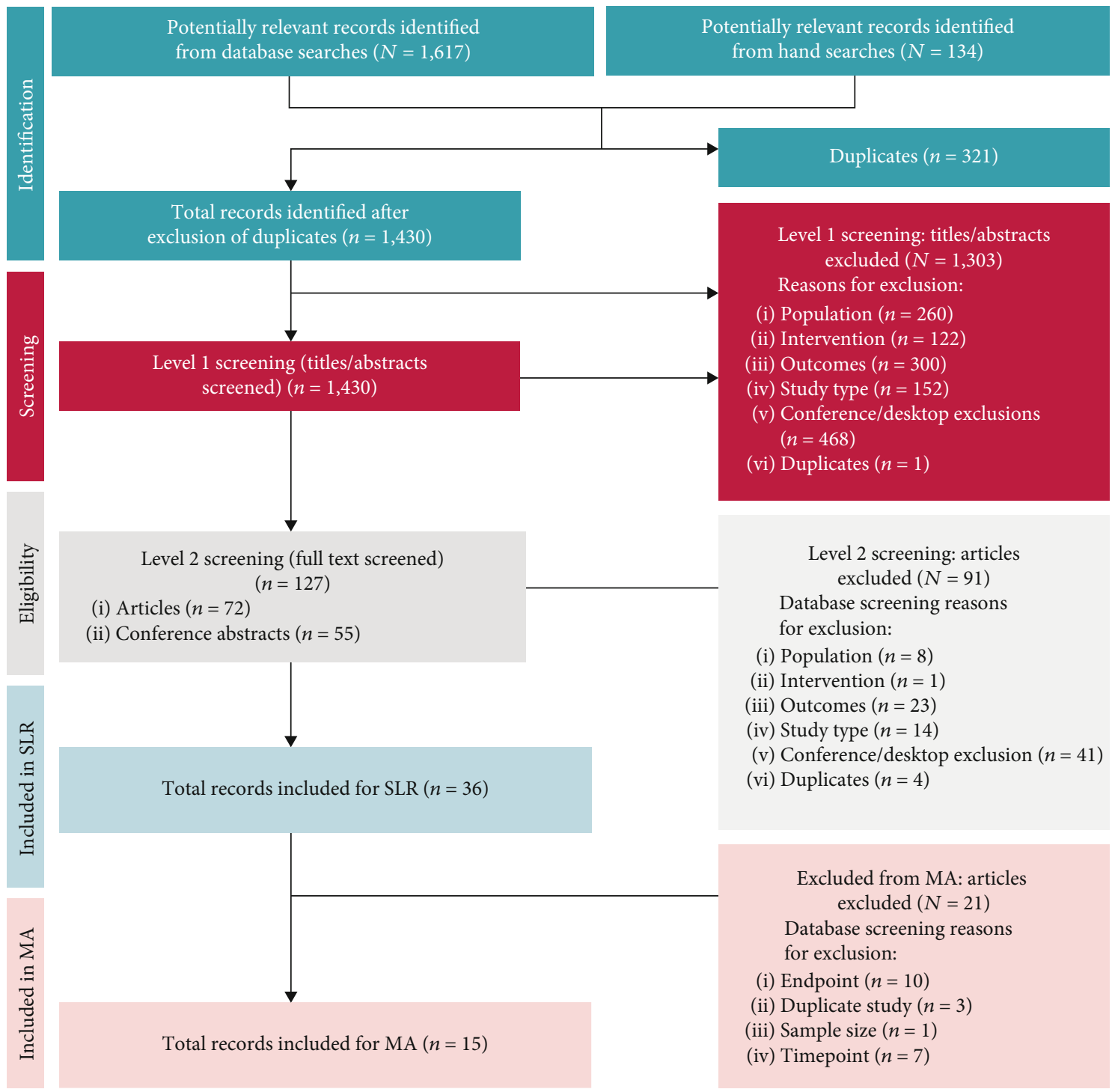

FIGURE 1: PRISMA flow diagram showing studies included in the systematic literature review (SLR) and meta-analysis (MA). PRISMA: Preferred Reporting Items for Systematic Reviews and Meta-Analyses.

cumulative rate of endoscopic remission with early- versus late-stage CD at 6 months: $29 \%(n=62)$ in patients with a disease duration of $\leq 2$ years, versus $13 \%(n=588)$ for disease duration of $>2$ years. A prospective cohort study, stratifying endoscopic remission rates by disease duration, reported a similar trend for higher rates of endoscopic remission in patients with shorter CD duration, which was more pronounced at week 52 versus week 26 of vedolizumab treatment [57]. This study also reported higher rates of endoscopic remission at 26 weeks in anti-TNF-naïve $(62 \%$ (95\% CI: $32 \%-85 \%) ; n=13$ ) versus anti-TNF-experienced patients (29\% (95\% CI: $20 \%-39 \%) ; n=97)$. Also, at 52 weeks, rates were $62 \%$ (95\% CI: $32 \%-85 \%)$ versus 33\% (95\% CI: 24\%-43\%) for anti-TNF-naïve versus anti-TNF-experienced patients, respectively [57].

3.2. Meta-analysis: MH Outcomes. Data from 15 separate real-world studies defining $\mathrm{MH}$ using absence of ulcers/ero- sions and/or SES-CD cut - points $<4$ were included for meta-analysis (Table 2); 21 publications were excluded primarily because of irrelevant time of measurement and not defining $\mathrm{MH}$ by absence of ulcers/erosions and/or SES-CD score of $\leq 4$ (Figure 1 and Supplementary Table 7).

Most (nine) of the 15 included studies were retrospective, five were prospective, and one was a registry-based study. All studies included a mix of anti-TNF-naïve and -experienced patients, apart from Kopylov [41], which included only antiTNF-naïve patients, and two studies for which this information was not available. Among studies with a mix of antiTNF- naive and -experienced patients, prior use of anti-TNF agents ranged from $68.9 \%$ to $99.4 \%$. Most studies used absence of ulcers and/or erosions (10 studies) or resolution of deep ulcers (one study) and/or SES-CD score of $<4$ (three studies), SES-CD score of $<2$ (one study), and SES-CD score of 0 (one study) (Table 2). The number of patients with endpoint data varied across the studies from 11 to 650; baseline 


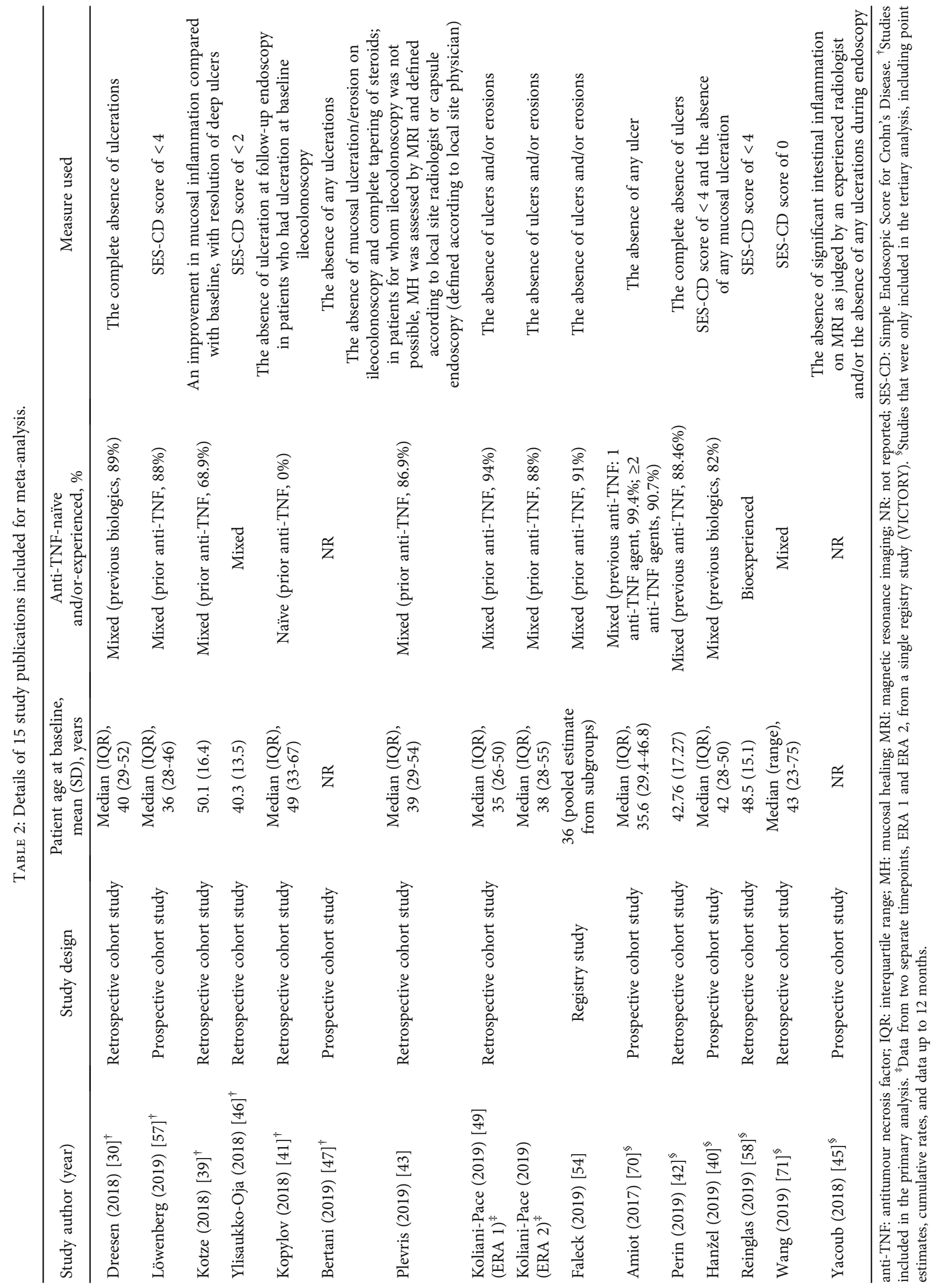


TABle 3: Patient baseline characteristics for studies pooled for meta-analysis.

\begin{tabular}{|c|c|c|}
\hline \multicolumn{2}{|l|}{ Baseline characteristics } & \multirow{2}{*}{$\frac{\text { No. of studies reporting data }}{14}$} \\
\hline $\operatorname{Age}^{\dagger}$ & $35-50.1$ years & \\
\hline Age at diagnosis ${ }^{*}$ & 22-32 years & 6 \\
\hline Male sex & $30 \%-64 \%$ & 14 \\
\hline Disease duration $^{\S}$ & 8-19.5 years & 10 \\
\hline Active smokers & $8.8 \%-30 \%$ & 12 \\
\hline Disease location & $\begin{array}{l}\text { (i) Ileal: } 0 \%-35.9 \% \\
\text { (ii) Colonic: } 14 \%-31.9 \% \\
\text { (iii) Ileocolonic: } 46 \%-86 \% \\
\text { (iv) Upper GI: } 1.3 \%-11.1 \%\end{array}$ & 11 \\
\hline Disease behaviour & $\begin{array}{l}\text { (i) Stricturing disease: } 21.3 \%-41.7 \% \\
\text { (ii) Penetrating disease: } 12 \%-25 \% \\
\text { (iii) Perianal disease: } 12 \%-45.3 \%\end{array}$ & 14 \\
\hline Extraintestinal manifestation & $27 \%-31.9 \%$ & 3 \\
\hline Crohn's disease-related surgery & $36 \%-49.1 \%$ & 7 \\
\hline Concomitant treatment & $\begin{array}{l}\text { (i) Corticosteroids } \\
\text { (ii) Immunomodulators }\end{array}$ & 14 \\
\hline Prior medication usage & & 14 \\
\hline Steroids & $34 \%-41 \%$ & 2 \\
\hline Immunomodulators & $76.2 \%-97.5 \%$ & 2 \\
\hline Biologics & $\begin{array}{l}\text { (i) Infliximab }(n=3): 54.0 \%-75.0 \% \\
\text { (ii) Adalimumab }(n=3): 52.0 \%-68.0 \% \\
\text { (iii) Golimumab }(n=2): 0 \\
\text { (iv) Ustekinumab }(n=1): 17 \% \\
\text { (v) Anti-TNF agent }(n=11): 0 \%-100 \%\end{array}$ & 14 \\
\hline Mixed anti-TNF-naïve and -experienced patients & Prior anti-TNF agents: $68.9 \%-99.4 \%$ & 10 \\
\hline Anti-TNF-experienced patients only & & 1 \\
\hline Anti-TNF-naïve patients only & & 1 \\
\hline Mucosal healing definitions & Absence of ulcers/erosions & 10 \\
\hline & Resolution of deep ulcers & 1 \\
\hline & And/or SES-CD score of $<4$ & 3 \\
\hline & And/or SES-CD score of $<2$ & 1 \\
\hline & And/or SES-CD score of 0 & 1 \\
\hline
\end{tabular}

anti-TNF: antitumour necrosis factor; GI: gastrointestinal; SES-CD: Simple Endoscopic Score for Crohn's Disease. ${ }^{\dagger}$ Four studies (Kotze et al. [39]; YlisaukkoOja et al. [46]; Perin et al. [42]; Reinglas et al. [58]) reported mean age and standard deviation. ${ }^{*}$ Three studies (Kotze et al. [39]; Amiot et al. [70]; Wang et al. [71]) reported mean age at diagnosis and standard deviation. ${ }^{\$}$ Two studies (Kotze et al. [39]; Ylisaukko-Oja et al. [46]) reported mean disease duration and standard deviation. ${ }^{9}$ Patients who were steroid dependent or steroid refractory at baseline.

characteristics are summarised in Table 3. For the 6-month meta-analysis, sex ratios differed slightly across studies, from $30 \%$ to $54 \%$ of males, and median age ranged from 30 to 56.1 years.

3.3. Pooled Estimates for Rates of $M H$ from Meta-analysis. In the primary analysis, pooled $\mathrm{MH}$ rates considering point estimates were $31.8 \%$ at 6 months $(n=223$, five studies) (Figure $2(\mathrm{a})$ ) and $33.4 \%$ at 12 months $(n=151$, three studies) (Figure 2(b)). There was no evidence of heterogeneity: $I^{2}=0 \%$ in both the 6- and 12-month analyses.

In the secondary analysis, which combined point estimates and cumulative rates in a single analysis, pooled $\mathrm{MH}$ rates were $24.5 \%$ within 6 months ( $n=1,013$, seven studies) (Figure 3(a)) and $40.5 \%$ within 12 months $(n=941$, five studies) (Figure 3(b)). There was considerable heterogeneity in the 6-month $\left(I^{2}=85 \%\right)$ and 12 -month results $\left(I^{2}=86 \%\right)$.
In the tertiary analysis, the pooled $\mathrm{MH}$ rate at 12 months was $41.5 \%$ (95\% CI: 33.7\%-49.4\%); however, considerable heterogeneity was observed $\left(I^{2}=82 \%\right)$.

\section{Discussion}

This comprehensive review of the real-world effect of vedolizumab on $\mathrm{MH}$ in patients with $\mathrm{CD}$ identified 36 studies reporting $\mathrm{MH}$-related outcomes published in the last 7 years. Rates for $\mathrm{MH}$-related endpoints, including $\mathrm{MH}$, endoscopic remission, endoscopic response, deep remission, and endoscopic healing, were frequently within the 26\%-50\% range. $\mathrm{MH}$ rates in vedolizumab-treated patients were similar, despite considerable cross-study variability, suggesting generalisability of the evidence. In the primary meta-analysis, rates of $\mathrm{MH}$ were consistent with pooled estimates of 


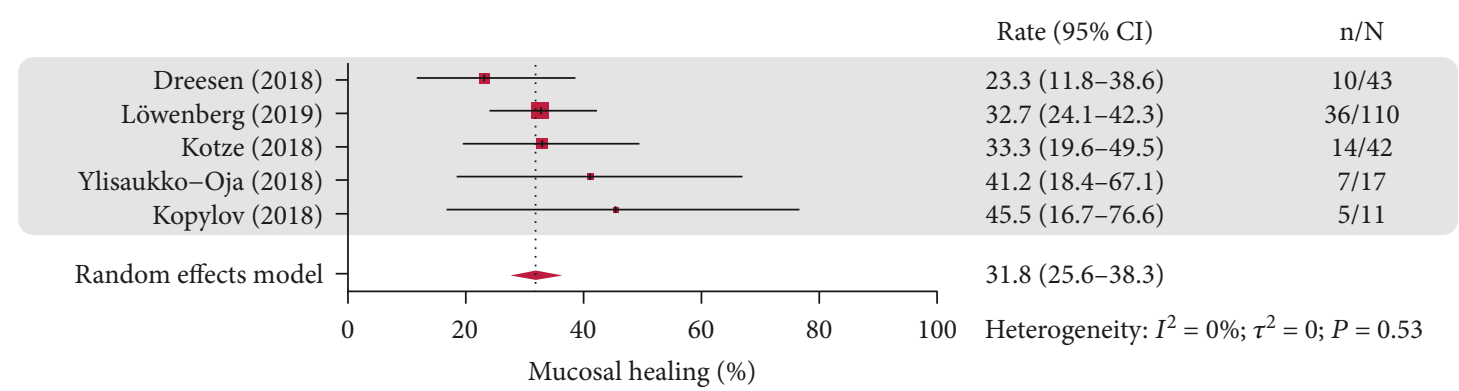

(a) Mucosal healing at 6 months: point estimates

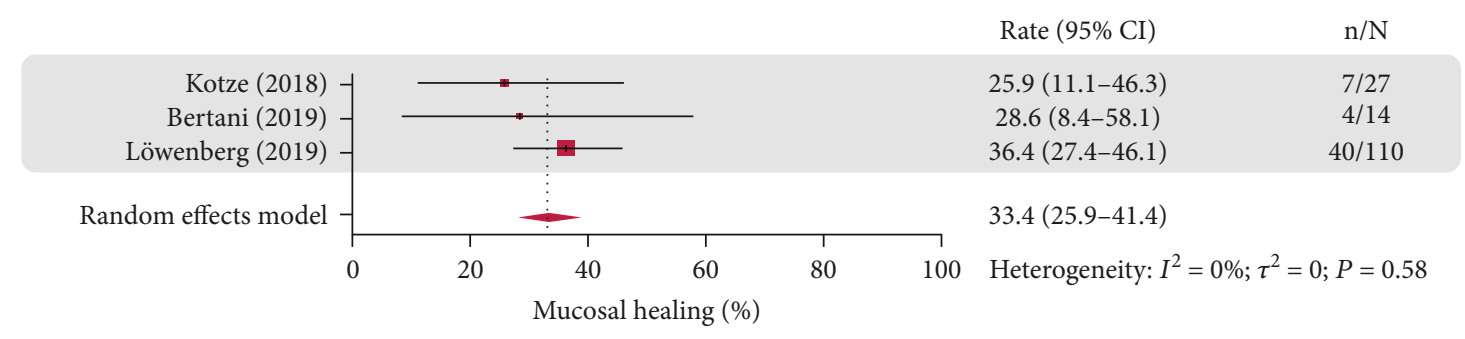

(b) Mucosal healing at 12 months: point estimates

FIgUre 2: Meta-analysis results: primary analysis. The 6-month analysis (a) included data reported from 22 to 26 weeks. The 12 -month analysis (b) included data reported from 52 to 54 weeks. Endpoint definitions: absence of ulcers/erosions and/or Simple Endoscopic Score for Crohn's Disease cut - points $<4$ (Table 2). CI: confidence interval.

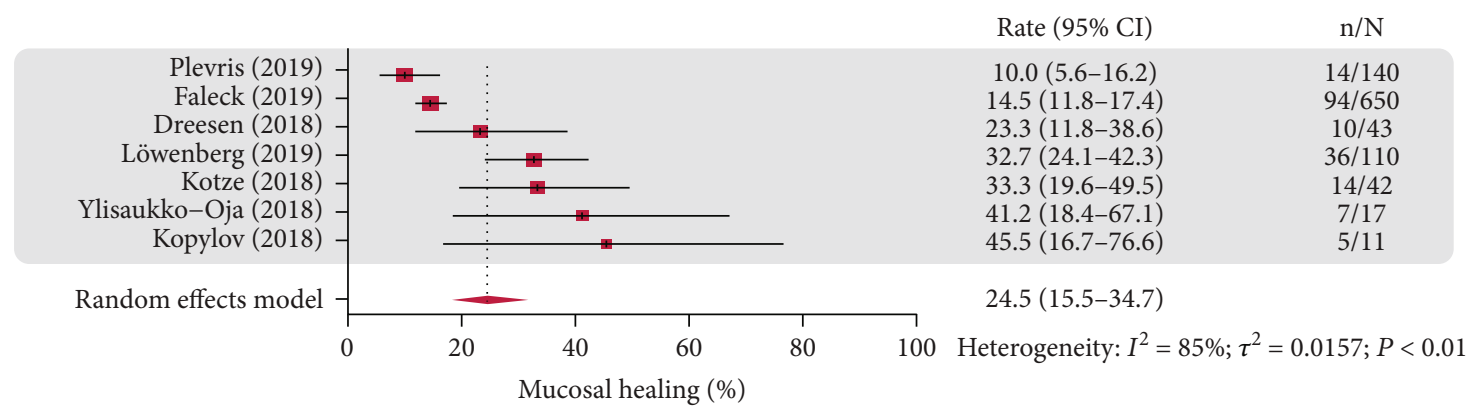

(a) Mucosal healing at 6 months: point estimates and cumulative rates

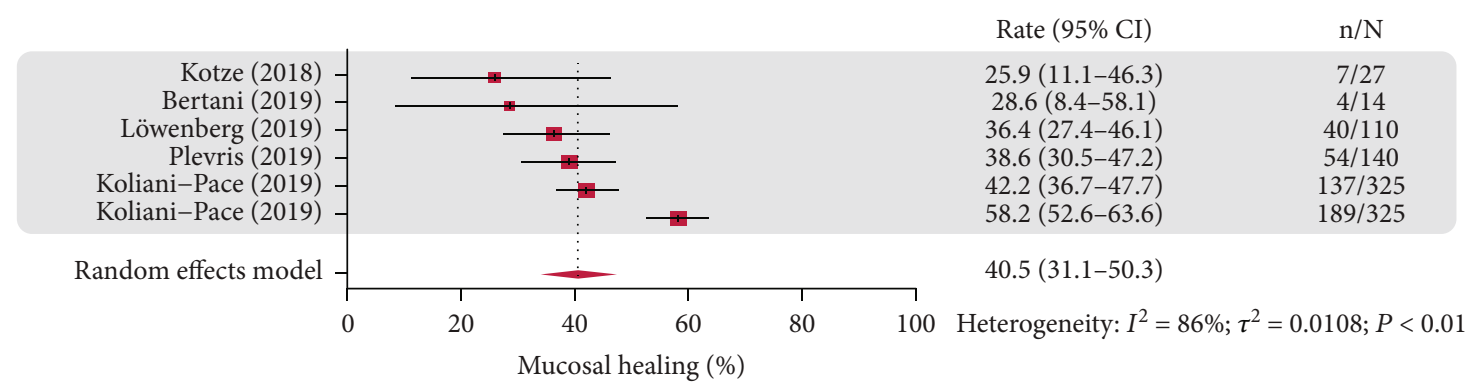

(b) Mucosal healing at 12 months: point estimates and cumulative rates

FIGURE 3: Meta-analysis results: secondary analysis. Faleck [54], Plevris [43], and Koliani-Pace [49] reported cumulative rates; other studies reported point estimates. The 6-month analysis (a) included data reported from 22 to 30 weeks. The Plevris [43] and Faleck [54] studies, reporting cumulative rates, appeared to be outliers. The 12-month analysis (b) included data reported from 52 weeks to 13.5 months. Cumulative rates from ERA 2 of the VICTORY trial reported by Koliani-Pace [49] were higher than those observed for other studies included in this meta-analysis. Endpoint definitions: absence of ulcers/erosions and/or Simple Endoscopic Score for Crohn's Disease cut - points $<4$ (Table 2). CI: confidence interval.

approximately one-third of vedolizumab-treated patients with $\mathrm{CD}$ achieving $\mathrm{MH}$ at 6 and 12 months.

Real-world effectiveness data for $\mathrm{MH}$ corroborate the findings from clinical trials reporting $\mathrm{MH}$ efficacy in vedolizumab-treated patients with CD $[18,33]$. In the VERSIFY phase $3 \mathrm{~b}$ clinical trial, rates of endoscopic remission (SES-CD score of $\leq 4)$ and endoscopic response $(\geq 50 \%$ reduction in baseline SES-CD score) at 52 weeks $(n=101)$ 
were $17.9 \%$ and $53.6 \%$, respectively [18]. Additionally, a retrospective analysis of data from 24 patients from Leuven University Hospitals enrolled in the GEMINI LTS study (who received vedolizumab on a 4-weekly dosing schedule for $\geq 1$ year) noted durable $\mathrm{MH}$ (absence of ulceration) in $29 \%$ of patients with CD (median (range) time on treatment 33 (6-59) months) [33]. The lower $\mathrm{MH}$ rates reported in clinical trials versus real-world settings might be due to protocol and patient population differences, endoscopic assessment by central reviewers, stricter definitions of $\mathrm{MH}$ applied to a smaller, selected patient sample, and protocol restrictions on treatment optimisation. The prospective, open-label LOVE-CD study of 110 patients (included in this meta-analysis of real-world evidence) used blinded evaluation of SES-CD scores by central reviewers (MH defined as SES-CD score of $<4$ ) but reported higher rates of endoscopic remission (33\% and 36\%) and endoscopic responses (40\% and $46 \%$ ) at 6 and 12 months of treatment, respectively. The difference may be due, in part, to the additional vedolizumab infusion at week 10 permitted by the protocol in LOVE-CD [57].

The treat-to-target strategy of early immunosuppression combined with tight and frequent control of mucosal inflammation was developed with the aim of reducing the risk of development of debilitating comorbidities in CD [5, 8]. Complete MH (SES-CD score of 0 ) achieved 2 years after immunosuppressive therapy in treatment-naïve patients has been associated with higher rates of 4-year steroid-free remission [11, 59]. Consistent with these findings, realworld vedolizumab studies also indicated that patients with shorter versus longer disease durations had higher endoscopic remission rates $[54,57]$. In real-world studies from this SLR [28, 49, 57] and in VERSIFY [18], rates for $\mathrm{MH}$ and endoscopic remission in vedolizumab-treated patients were also higher among anti-TNF-naïve patients versus those previously treated with anti-TNF agents. This clinical effect of prior anti-TNF treatment on the subsequent efficacy of vedolizumab could be explained by the observation that anti-TNF biologics downregulate the expression of the mucosal addressin cell adhesion molecule-1 (MAdCAM-1), the primary ligand of the $\alpha_{4} \beta_{7}$ integrin heterodimer found on subsets of peripheral lymphocytes [60].

Considering vedolizumab treatment relative to other biologic treatments for $\mathrm{CD}$, data from the EVOLVE study demonstrated comparable real-world $\mathrm{MH}$ rates in biologicnaïve patients with moderate to severe $\mathrm{CD}$ treated with vedolizumab or anti-TNF agents [28, 61]. Cumulative $\mathrm{MH}$ rates at 12 months (62.5\% vs. 59.7\%, respectively) and over 24 months (100.0\% vs. $90.1 \%$, respectively) included in this SLR were comparable between vedolizumab-treated and anti-TNF-treated patients [28]. These high rates were based on the cumulative number of at-risk patients in each treatment group, calculated by computing the probability of $\mathrm{MH}$. As such, outcome rates of $>90 \%$ do not correspond to $90 \%$ or $100 \%$ of all CD patients achieving this outcome. Our study shows that most patients achieve $\mathrm{MH}$ within 6 months, and the rate is stable at 1 year without further significant increases. In the context of the treat-to-target strategy, this would support optimisation of treatment if $\mathrm{MH}$ is not achieved within 6 months with vedolizumab. The combined use of biomarkers and clinical indices, as well as the measurement of drug levels, could help improve clinical outcomes, including $\mathrm{MH}$ [62-64].

Real-world evidence can be used to validate clinical trials results [65]; the US Food and Drug Administration has created a framework for evaluating real-world evidence to help support study requirements after treatments have been approved [66]. Data from clinical practice are more reflective of the heterogeneity in patient characteristics and drug exposure/adherence than controlled trials $[65,67,68]$. However, the lack of standardised information across real-world studies is a limitation that can decrease comparability and potentially introduce bias. Here, we found high variability in terminology and specific definition/cut-off values for $\mathrm{MH}$ related endpoints. Restricting the primary meta-analysis to studies that measured $\mathrm{MH}$ using absence of ulcers/erosions and/or SES-CD cut - points $<4$ was designed to minimise this effect on pooled analysis of $\mathrm{MH}$ rates.

Three types of analysis for point estimates were conducted to assess the variability of treatment effect across different study subgroupings [69]. These analyses did not indicate a large variability in treatment effect size when selecting different subsets of patients based on length of follow-up or cumulative rates versus point estimates. For the primary analysis, no significant evidence of heterogeneity was observed in the results across studies, although few studies were included (five and three studies for the 6month and 12-month analyses, respectively). One study included in the primary analysis had a low number of patients $(n=11)$. For the secondary meta-analysis of point estimates and cumulative rates, considerable heterogeneity measured by $I^{2}$ was observed, suggesting that the pooled estimates should be interpreted with caution.

The lack of central reading for endoscopy data is also a limitation of pooled $\mathrm{MH}$ rates from independent realworld studies; however, most studies were from wellknown and expert institutions. The National Institutes of Health quality assessment indicated that all studies included in this SLR and meta-analysis were of fair quality (Supplementary Table 5). Many studies reported MH outcomes for only a portion of the total enrolled patients because of the lack of endoscopic assessment data; therefore, the sample sizes for $\mathrm{MH}$ and endoscopic remission results were small, and the baseline characteristics of these patients were mostly unavailable.

$\mathrm{MH}$ has been identified as an important treatment goal in recent $\mathrm{CD}$ management guidelines [8], associated with long-term benefits for patients [10-14]. More comparative treatment effectiveness data using standardised, wellvalidated $\mathrm{MH}$ endpoints would help inform risk-benefit evaluations for CD therapies [4]; however, a more pressing challenge for the management of CD may lie in maintaining sustainable treatment effects over the disease course.

\section{Conclusions}

The findings of this SLR support the effectiveness of vedolizumab for achieving $\mathrm{MH}$ in patients with $\mathrm{CD}$ treated in real- 
world clinical settings. In a meta-analysis of pooled data from real-world studies, approximately one-third of vedolizumab-treated patients with $\mathrm{CD}$ achieved $\mathrm{MH}$ at 6 and 12 months, despite utilisation in largely biologicrefractory patients. Indications that $\mathrm{MH}$ rates may be higher in anti-TNF-naïve versus -experienced patients, and the potential benefit of early vedolizumab treatment, warrant further investigation.

\section{Data Availability}

The datasets, including the redacted study protocol, redacted statistical analysis plan, and individual participants data supporting the results reported in this article, will be made available within three months from initial request, to researchers who provide a methodologically sound proposal. The data will be provided after its deidentification, in compliance with applicable privacy laws, data protection, and requirements for consent and anonymization. Data are available upon request via application at https://search.vivli.org.

\section{Ethical Approval}

The authors confirm that the ethical policies of the journal, as noted on the journal's author guidelines page, have been adhered to. No ethical approval was required as this is a review article with no original research data.

\section{Disclosure}

This work is presented as an ePoster at Digestive Disease Week ${ }^{\circledR}$ Gastroenterology 2021:160:S-702-3.

\section{Conflicts of Interest}

S.D. reports lecture/consulting fees from AbbVie, Allergan, Biogen, Boehringer Ingelheim, Celgene, Celltrion, Ferring, Hospira, Johnson \& Johnson, Merck, Merck Sharp \& Dohme, Mundipharma, Pfizer, Sandoz, Takeda, TiGenix, UCB, and Vifor. P.K. and C.A. are employees of and hold stock/stock options in Takeda. SW is a former employee of and holds stock/stock options in Takeda. J.Y., J.-G.L.M., S.K., and E.H. are employees of RTI Health Solutions, which received funding from Takeda. P.M.I. reports lecture fees from AbbVie, Bristol Myers Squibb, Celgene, Falk Pharma, Ferring, Gilead, Janssen, Merck Sharp \& Dohme, Pfizer, Sandoz, Sapphire Medical, Shire, Takeda, Tillotts, and Warner Chilcott; financial support for research from Merck Sharp \& Dohme, Pfizer, and Takeda; and advisory fees from AbbVie, Arena, Genentech, Gilead, Hospira, Janssen, Lilly, Merck Sharp \& Dohme, Pfizer, Pharmacosmos, Procise, Prometheus, Roche, Samsung Bioepis, Sandoz, Takeda, Topivert, VH2, Vifor, and Warner Chilcott.

\section{Authors' Contributions}

P.K., J.Y., J.-G.L.M., S.K., E.H., and S.W. contributed to the conception and design of the study. J.Y., J.-G.L.M., S.K., E.H., and C.A. contributed to the acquisition of the data. S.D., P.K., J.Y., J.-G.L.M., S.K., E.H., C.A., S.W., and P.M.I. contributed to the analysis and interpretation of the data. S.D., P.K., J.Y., J.-G.L.M., S.K., E.H., C.A., S.W., and P.M.I. were involved in drafting the article and/or revising it critically for intellectual content. All authors approved the final version of the article, including the authorship list.

\section{Acknowledgments}

This study was sponsored by Takeda. The sponsor was involved in study design, data analysis and interpretation, and writing of the manuscript. Medical writing support was provided by Isobel Lever, $\mathrm{PhD}$, of Excel Medical Affairs, funded by Takeda.

\section{Supplementary Materials}

Supplementary Table 1: MEDLINE literature search strategy for real-world evidence of mucosal healing with vedolizumab. Supplementary Table 2: EMBASE literature search strategy for real-world evidence of mucosal healing with vedolizumab. Supplementary Table 3: Cochrane literature search strategy for real-world evidence of mucosal healing with vedolizumab. Supplementary Table 4: EMBASE conference abstracts: literature search strategy for real-world evidence of mucosal healing with vedolizumab. Supplementary Table 5: Quality assessment of studies using NIH QA cohort study tool A. Supplementary Table 6: Patient baseline characteristics for the 36 studies identified in the systematic literature review. Supplementary Table 7: Studies excluded from MA. Supplementary materials references list. PRISMA Checklist. (Supplementary Materials)

\section{References}

[1] K. Cushing and P. D. R. Higgins, "Management of Crohn Disease: a Review," Journal of the American Medical Association, vol. 325, no. 1, pp. 69-80, 2021.

[2] J. Torres, S. Mehandru, J. F. Colombel, and L. Peyrin-Biroulet, "Crohn's disease," Lancet, vol. 389, pp. 1741-1755, 2017.

[3] B. Pariente, J. Y. Mary, S. Danese et al., "Development of the Lémann index to assess digestive tract damage in patients with Crohn's disease," Gastroenterology, vol. 148, pp. 52-63.e3, 2015.

[4] J. F. Colombel, G. D'Haens, W. J. Lee, J. Petersson, and R. Panaccione, "Outcomes and strategies to support a treatto-target approach in inflammatory bowel disease: a systematic review," Journal of Crohn's \& Colitis, vol. 14, no. 2, pp. 254-266, 2020.

[5] E. Klenske, C. Bojarski, M. Waldner, T. Rath, M. F. Neurath, and R. Atreya, "Targeting mucosal healing in Crohn's disease: what the clinician needs to know," Therapeutic Advances in Gastroenterology, vol. 12, 2019.

[6] R. C. Ungaro, S. Aggarwal, O. Topaloglu, W. J. Lee, R. Clark, and J. F. Colombel, "Systematic review and meta-analysis: efficacy and safety of early biologic treatment in adult and paediatric patients with Crohn's disease," Alimentary Pharmacology \& Therapeutics, vol. 51, pp. 831-842, 2020.

[7] C. Ma, B. D. van Rhijn, V. Jairath et al., "Heterogeneity in clinical, endoscopic, and histologic outcome measures and placebo response rates in clinical trials of eosinophilic esophagitis: a 
systematic review," Clinical Gastroenterology and Hepatology, vol. 16, pp. 1714-1729.e3, 2018.

[8] D. Turner, A. Ricciuto, A. Lewis et al., "STRIDE-II: An Update on the Selecting Therapeutic Targets in Inflammatory Bowel Disease (STRIDE) Initiative of the International Organization for the Study of IBD (IOIBD): Determining Therapeutic Goals for Treat-to-Target strategies in IBD," Gastroenterology, vol. 160, no. 5, pp. 1570-1583, 2021.

[9] J. S. Lee, E. S. Kim, and W. Moon, "Chronological review of endoscopic indices in inflammatory bowel disease," Clinical endoscopy, vol. 52, pp. 129-136, 2019.

[10] M. F. Neurath and S. P. Travis, "Mucosal healing in inflammatory bowel diseases: a systematic review," Gut, vol. 61, no. 11, pp. 1619-1635, 2012.

[11] F. Baert, L. Moortgat, G. Van Assche et al., "Mucosal Healing Predicts Sustained Clinical Remission in Patients With EarlyStage Crohn's Disease," Gastroenterology, vol. 138, no. 2, pp. 463-468, 2010.

[12] I. Alfaro, M. C. Masamunt, N. Planell et al., "Endoscopic response to tumor necrosis factor inhibitors predicts long term benefits in Crohn's disease," World Journal of Gastroenterology, vol. 25, pp. 1764-1774, 2019.

[13] P. Rutgeerts, S. Vermeire, and G. Van Assche, "Mucosal healing in inflammatory bowel disease: impossible ideal or therapeutic target?," Gut, vol. 56, pp. 453-455, 2007.

[14] W. J. Sandborn, S. Hanauer, G. Van Assche et al., "Treating beyond symptoms with a view to improving patient outcomes in inflammatory bowel diseases," Journal of Crohn's \& Colitis, vol. 8, pp. 927-935, 2014.

[15] S. C. Shah, J. F. Colombel, B. E. Sands, and N. Narula, "Systematic review with meta-analysis: mucosal healing is associated with improved long-term outcomes in Crohn's disease," Alimentary Pharmacology \& Therapeutics, vol. 43, pp. 317-333, 2016.

[16] G. R. Lichtenstein, E. V. Loftus, K. L. Isaacs, M. D. Regueiro, L. B. Gerson, and B. E. Sands, "ACG Clinical Guideline: Management of Crohn's disease in adults," The American Journal of Gastroenterology, vol. 113, pp. 481-517, 2018.

[17] F. Gomollón, A. Dignass, V. Annese et al., "3rd European evidence-based consensus on the diagnosis and management of Crohn's disease 2016: part 1: diagnosis and medical management," Journal of Crohn's \& Colitis, vol. 11, pp. 3-25, 2017.

[18] S. Danese, W. J. Sandborn, J. F. Colombel et al., "Endoscopic, radiologic, and histologic healing with vedolizumab in patients with active Crohn's disease," Gastroenterology, vol. 157, pp. 1007-1018.e7, 2019.

[19] L. Peyrin-Biroulet, W. Reinisch, J. F. Colombel et al., "Clinical disease activity, C-reactive protein normalisation and mucosal healing in Crohn's disease in the SONIC trial," Gut, vol. 63, pp. 88-95, 2014.

[20] L. Laterza, A. C. Piscaglia, L. M. Minordi et al., "Multiparametric evaluation predicts different mid-term outcomes in Crohn's disease," Digestive Diseases, vol. 36, pp. 184-193, 2018.

[21] J. Jones, E. V. Loftus Jr., R. Panaccione et al., "Relationships between disease activity and serum and fecal biomarkers in patients with Crohn's disease," Clinical Gastroenterology and Hepatology, vol. 6, pp. 1218-1224, 2008.

[22] J. D. Lewis, P. Rutgeerts, B. G. Feagan et al., "Correlation of stool frequency and abdominal pain measures with Simple Endoscopic Score for Crohn's disease," Inflammatory Bowel Diseases, vol. 26, pp. 304-313, 2020.
[23] Entyvio (vedolizumab) Prescribing Information, Takeda Pharmaceuticals America Inc, Deerfield, IL, USA, 2021, https:// www.accessdata.fda.gov/drugsatfda_docs/label/2021/ 125476s038s039lbl.pdf.

[24] Entyvio INN-vedolizumab Summary of Product Characteristics, Takeda Pharma A/S, Taastruup, Denmark, 2021, https:// www.ema.europa.eu/en/documents/product-information/ entyvio-epar-product-information_en.pdf.

[25] T. Wyant, E. Fedyk, and B. Abhyankar, "An overview of the mechanism of action of the monoclonal antibody vedolizumab," Journal of Crohn's \& Colitis, vol. 10, pp. 1437-1444, 2016.

[26] W. J. Sandborn, B. G. Feagan, P. Rutgeerts et al., "Vedolizumab as Induction and Maintenance Therapy for Crohn's Disease," The New England Journal of Medicine, vol. 369, no. 8, pp. 711-721, 2013.

[27] B. E. Sands, W. J. Sandborn, G. Van Assche et al., "Vedolizumab as induction and maintenance therapy for Crohn's disease in patients Naïve to or who have failed tumor necrosis factor antagonist therapy," Inflammatory Bowel Diseases, vol. 23, no. 1, pp. 97-106, 2017.

[28] B. Bressler, G. Mantzaris, M. Silverberg et al., "P 621 real-world effectiveness and safety of vedolizumab and anti-TNF in biologic-naive Crohn's disease patients: results from the EVOLVE study," Journal of Crohn's \& Colitis, vol. 13, Supplement_1, pp. S427-S428, 2019.

[29] B. Christensen, R. J. Colman, D. Micic et al., "Vedolizumab as induction and maintenance for inflammatory bowel disease: 12-month effectiveness and safety," Inflammatory Bowel Diseases, vol. 24, pp. 849-860, 2018.

[30] E. Dreesen, B. Verstockt, S. Bian et al., "Evidence to support monitoring of vedolizumab trough concentrations in patients with inflammatory bowel diseases," Clinical Gastroenterology and Hepatology, vol. 16, pp. 1937-1946.e8, 2018.

[31] P. S. Dulai, S. Singh, X. Jiang et al., "The real-world effectiveness and safety of vedolizumab for moderate-severe Crohn's disease: results from the US VICTORY consortium," The American Journal of Gastroenterology, vol. 111, pp. 1147$1155,2016$.

[32] U. Kopylov, I. Avni-Biron, Y. Ron et al., "Effectiveness and safety of vedolizumab for maintenance treatment in inflammatory bowel disease-the Israeli real world experience," Digestive and Liver Disease, vol. 51, pp. 68-74, 2019.

[33] M. Noman, M. Ferrante, R. Bisschops et al., "Vedolizumab induces long-term mucosal healing in patients with Crohn's disease and ulcerative colitis," Journal of Crohn's \& Colitis, vol. 11, pp. 1085-1089, 2017.

[34] D. Moher, A. Liberati, J. Tetzlaff, and D. G. Altman, "Preferred reporting items for systematic reviews and meta-analyses: the PRISMA statement," Journal of Clinical Epidemiology, vol. 62, no. 10, pp. 1006-1012, 2009.

[35] J. P. Higgins, J. Thomas, J. Chandler, M. Cumpston, T. Li, M. J. Page, and V. A. Welch, Eds., Cochrane Handbook for Systematic Reviews of Interventions. Version 6.2 (updated February 2021): Cochrane, John Wiley \& Sons, 2021.

[36] Quality assessment tool for observational cohort and crosssectional studies, National Institutes of Health. National Heart, Lung, and Blood Institute, 2021, https://www.nhlbi.nih.gov/ health-topics/study-quality-assessment-tools.

[37] R. Bor, A. Fábián, M. Matuz et al., "Real-life efficacy of vedolizumab on endoscopic healing in inflammatory bowel disease - 
a nationwide Hungarian cohort study," Expert Opinion on Biological Therapy, vol. 20, pp. 205-213, 2020.

[38] R. Bor, F. Klaudia, P. Miheller et al., "DOP007 efficacy of vedolizumab induction therapy on clinical and endoscopic activity in patients with anti-tumour necrosis factor alpha-resistant inflammatory bowel disease," Journal of Crohn's \& Colitis, vol. 12, supplement_1, pp. S034-S035, 2018.

[39] P. G. Kotze, C. Ma, A. Almutairdi et al., "Real-world clinical, endoscopic and radiographic efficacy of vedolizumab for the treatment of inflammatory bowel disease," Alimentary Pharmacology \& Therapeutics, vol. 48, pp. 626-637, 2018.

[40] J. Hanžel, N. Sever, I. Ferkolj et al., "Early vedolizumab trough levels predict combined endoscopic and clinical remission in inflammatory bowel disease," United European Gastroenterology Journal, vol. 7, pp. 741-749, 2019.

[41] U. Kopylov, B. Verstockt, L. Biedermann et al., "Effectiveness and safety of vedolizumab in Anti-TNF-Naïve patients with inflammatory bowel disease-a multicenter retrospective European study," Inflammatory Bowel Diseases, vol. 24, no. 11, pp. 2442-2451, 2018.

[42] R. L. Perin, A. O. Damião, C. Flores et al., "Vedolizumab in the management of inflammatory bowel diseases: a Brazilian observational multicentric study," Arquivos de Gastroenterologia, vol. 56, no. 3, pp. 312-317, 2019.

[43] N. Plevris, C. S. Chuah, R. M. Allen et al., "Real-world effectiveness and safety of vedolizumab for the treatment of inflammatory bowel disease: the Scottish vedolizumab cohort," Journal of Crohn's \& Colitis, vol. 13, pp. 1111-1120, 2019.

[44] E. E. Vivio, N. Kanuri, J. J. Gilbertsen et al., "Vedolizumab effectiveness and safety over the first year of use in an IBD clinical practice," Journal of Crohn's \& Colitis, vol. 10, pp. 402409, 2016.

[45] W. Yacoub, N. Williet, L. Pouillon et al., "Early vedolizumab trough levels predict mucosal healing in inflammatory bowel disease: a multicentre prospective observational study," Alimentary Pharmacology \& Therapeutics, vol. 47, pp. 906-912, 2018.

[46] T. Ylisaukko-Oja, J. Aaltonen, H. Nuutinen et al., "High treatment persistence rate and significant endoscopic healing among real-life patients treated with vedolizumab - a Finnish Nationwide Inflammatory Bowel Disease Cohort Study (FINVEDO)," Scandinavian Journal of Gastroenterology, vol. 53, pp. 158-167, 2018.

[47] L. Bertani, L. Ceccarelli, M. G. Mumolo et al., "P416 Faecal calprotectin can predict mucosal healing in patients with inflammatory bowel diseases treated with vedolizumab: a prospective single-centre study," Journal of Crohn's \& Colitis, vol. 13, Supplement_1, pp. S317-S318, 2019.

[48] R. Bor, K. Farkas, P. Miheller et al., "P1810 short- and longterm efficacy of vedolizumab therapy on clinical and endoscopic activity in patients with anti-tumor necrosis factor alpha resistant inflammatory bowel disease," United European Gastroenterology Journal, vol. 7, no. S8, p. 916, 2019.

[49] J. L. Koliani-Pace, S. Singh, M. Luo et al., "Changes in vedolizumab utilization across US academic centers and community practice are associated with improved effectiveness and disease outcomes," Inflammatory Bowel Diseases, vol. 25, pp. 18541861, 2019.

[50] C. Quatraccioni, G. Annulli, L. M. Daretti et al., "P1105 vedolizumab induces deep remission and improves quality of life in patients with inflammatory bowel diseases: the ANCONA experience," United European Gastroenterology Journal, vol. 7, no. S8, p. 632, 2019.

[51] A. Tursi, G. Mocci, R. Faggiani et al., "Vedolizumab is effective and safe in real-life treatment of inflammatory bowel diseases outpatients: a multicenter, observational study in primary inflammatory bowel disease centers," European Journal of Internal Medicine, vol. 66, pp. 85-91, 2019.

[52] S. Bots, E. van Baalen, N. de Boer et al., "P376 clinical effectiveness of vedolizumab in a tertiary care cohort of IBD patients," Journal of Crohn's \& Colitis, vol. 12, supplement_1, pp. S294S295, 2018.

[53] G. Dragoni, S. Bagnoli, M. Le Grazie et al., "Long-term efficacy and safety of vedolizumab in patients with inflammatory bowel diseases: a real-life experience from a tertiary referral center," Journal of Digestive Diseases, vol. 20, pp. 235-242, 2019.

[54] D. M. Faleck, A. Winters, S. Chablaney et al., "Shorter disease duration is associated with higher rates of response to vedolizumab in patients with Crohn's disease but not ulcerative colitis," Clinical Gastroenterology and Hepatology, vol. 17, pp. 2497-2505.e1, 2019.

[55] A. T. M. Gan, W. P. W. Chan, K. L. Ling et al., "P634 realworld data on the efficacy and safety of vedolizumab therapy in patients with inflammatory bowel disease: a retrospective nation-wide cohort study in Singapore," Journal of Crohn's \& Colitis, vol. 13, Supplement_1, pp. S434-S435, 2019.

[56] L. Guidi, D. Pugliese, T. P. Tonucci et al., "Early vedolizumab trough levels predict treatment persistence over the first year in inflammatory bowel disease," United European Gastroenterology Journal, vol. 7, no. 9, pp. 1189-1197, 2019.

[57] M. Löwenberg, S. Vermeire, N. Mostafavi et al., "Vedolizumab induces endoscopic and histologic remission in patients with Crohn's disease," Gastroenterology, vol. 157, pp. 997-1006.e6, 2019.

[58] J. Reinglas, L. Gonczi, C. Verdon et al., "Low rate of drug discontinuation, frequent need for dose adjustment, and no association with development of new arthralgia in patients treated with vedolizumab: results from a tertiary referral IBD center," Digestive Diseases and Sciences, vol. 65, pp. 2046-2053, 2020.

[59] G. D'Haens, F. Baert, G. van Assche et al., "Early combined immunosuppression or conventional management in patients with newly diagnosed Crohn's disease: an open randomised trial," Lancet, vol. 371, no. 9613, pp. 660-667, 2008.

[60] P. Biancheri, A. Di Sabatino, L. Rovedatti et al., "Effect of tumor necrosis factor- $\alpha$ blockade on mucosal addressin celladhesion molecule-1 in Crohn's disease," Inflammatory Bowel Diseases, vol. 19, pp. 259-264, 2013.

[61] B. Bressler, A. Yarur, M. S. Silverberg et al., "Vedolizumab and Anti-Tumour Necrosis Factor $\alpha$ real-world outcomes in biologic-naïve inflammatory bowel disease patients: results from the EVOLVE study," Journal of Crohn's and Colitis, vol. 15, no. 10, pp. 1694-1706, 2021.

[62] B. Al-Bawardy, G. P. Ramos, M. A. Willrich et al., "Vedolizumab drug level correlation with clinical remission, biomarker normalization, and mucosal healing in inflammatory bowel disease," Inflammatory Bowel Diseases, vol. 25, pp. 580-586, 2019.

[63] S. Berends, M. Löwenberg, F. Baert et al., "DOP046 higher serum concentrations of vedolizumab are associated with superior endoscopic outcomes in Crohn's disease: data from the LOVE-CD trial," Journal of Crohn's \& Colitis, vol. 12, supplement_1, p. S063, 2018. 
[64] A. J. Yarur, P. Deepak, N. Vande Casteele et al., "Association between vedolizumab levels, anti-vedolizumab antibodies, and endoscopic healing index in a large population of patients with inflammatory bowel diseases," Digestive Diseases and Sciences, vol. 66, pp. 3563-3569, 2021.

[65] R. E. Sherman, S. A. Anderson, G. J. Dal et al., "Real-world evidence - what is it and what can it tell us?," The New England Journal of Medicine, vol. 375, pp. 2293-2297, 2016.

[66] Food and Drug Administration, "Real-world evidence," 2020, https://www.fda.gov/science-research/science-and-researchspecial-topics/real-world-evidence.

[67] S. de Lusignan, L. Crawford, and N. Munro, "Creating and using real-world evidence to answer questions about clinical effectiveness," BMJ Health \& Care Informatics, vol. 22, pp. 368-373, 2015.

[68] C. Ha, T. A. Ullman, C. A. Siegel, and A. Kornbluth, "Patients enrolled in randomized controlled trials do not represent the inflammatory bowel disease patient population," Clinical Gastroenterology and Hepatology, vol. 10, pp. 1002-1007, 2012.

[69] M. Richardson, P. Garner, and S. Donegan, "Interpretation of subgroup analyses in systematic reviews: a tutorial," Clinical Epidemiology and Global Health, vol. 7, no. 2, pp. 192-198, 2019.

[70] A. Amiot, M. Serrero, L. Peyrin-Biroulet et al., "One-year effectiveness and safety of vedolizumab therapy for inflammatory bowel disease: a prospective multicentre cohort study," Alimentary Pharmacology \& Therapeutics, vol. 46, no. 3, pp. 310-321, 2017.

[71] Y. Wang, J. Wang, J. Pekow et al., "Outcome of elective switching to vedolizumab in inflammatory bowel disease patients under tumor necrosis factor antagonist-maintained clinical remission," Journal of Gastroenterology and Hepatology, vol. 34, pp. 2090-2095, 2019. 